\title{
Cuffed Peritoneal Dialysis Catheter
}

National Cancer Institute

\section{Source}

National Cancer Institute. Cuffed Peritoneal Dialysis Catheter. NCI Thesaurus. Code C122619.

A hollow tube that is inserted in the abdomen to carry fluid into and out of the abdominal cavity where the exit site is secured just under the skin with a band-like structure to prevent slippage and infection. 